\title{
Robots take over electrophysiology
}

Image-guided targeting of fluorescently labeled neurons enables automated electrophysiological recordings of defined neuronal cell types.

Patch-clamp electrophysiology has been an invaluable technique in neuroscience; patch-clamp recordings from whole cells can provide a detailed picture of the electrophysiological properties of neurons. The technology involves approaching a cell with a glass micropipette, forming a seal on the membrane and breaking into the cell in order to establish an electrical continuum between the cell and the micropipette. Yet, since its invention in the seventies, this technique has remained labor intensive and continues to require experience and skill.

Automated approaches would help to increase throughput and to make the technique more widely accessible. Patch-clamp robots have been developed, but so far they have been blind to the cell types they record from. Two groups, led by Ed Boyden from the Massachusetts Institute of Technology and by Simon Schultz from the Imperial College London, have independently reported image-guided automated patchclamp techniques that make it possible to record from defined cells in the mouse brain in an automated fashion.

Some of the steps necessary for automated patch-clamp recording were established previously, such as the approach towards the target cell or the patching of random cells. But a key technical hurdle that had to be overcome for combining the individual achievements was tissue displacement during the approach of the micropipette. Both groups developed similar algorithms to compensate for tissue motion, and these algorithms work by acquiring the positions of the micropipette tip and the target cell using two-photon imagery. Once the pipette tip reaches the vicinity of the cell, the approach path is updated in order to compensate for tissue displacement caused by the pipette insertion. Thus, a closed-loop feedback system keeps the approaching micropipette on track. The following steps of seal formation and breaking in are similar to already established approaches that blindly targeted cells.

Boyden's group demonstrated the performance of their robot by recording from interneurons and pyramidal neurons in the

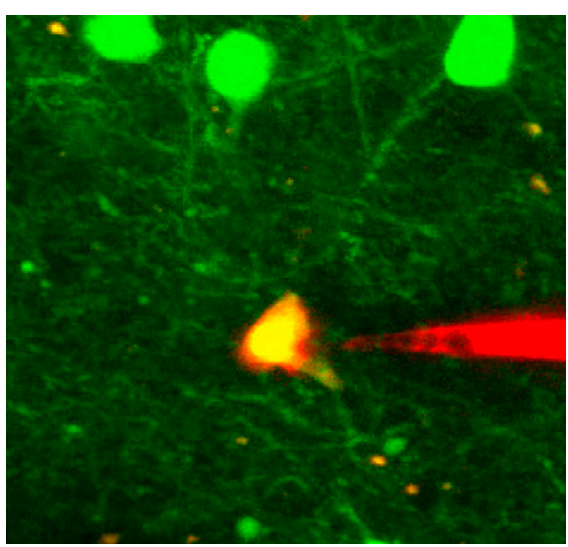

A micropipette (in red) in whole-cell patchclamp configuration during recording from an interneuron (in yellow) in the mouse visual cortex. Image reprinted with permission from Annecchino et al., Elsevier Inc.

mouse cortex (Suk et al., 2017). They successfully established a good seal on the cell in about $35 \%$ of the cases, and $60 \%$ of those cases resulted in a whole-cell patch-clamp configuration. Schultz's group targeted pyramidal neurons in the primary visual cortex in mice and successfully achieved a seal in roughly $47 \%$ of the cases, while they obtained a whole-cell patch-clamp recording in $48 \%$ of those (Annecchino et al., 2017). Thus, both robots achieved a similar overall success rate in about $20 \%$ of the trials, which is roughly comparable to the success rates of manual patch-clamp recording (although success depends on the skill level of the experimenter).

These image-guided patch-clamp robots are promising developments. However, they have so far only been applied in proof-of-principle experiments involving a limited number of trials. Their broader adoption will depend on their robustness and also on further developments, such as the automated loading of fresh micropipettes. Furthermore, experiments that require recording from two or more cells at the same time would benefit if the robotic capabilities could be multiplexed.

\section{Nina Vogt}

\section{RESEARCH PAPERS}

Suk, H.-J. et al. Closed-loop real-time imaging enables fully automated cell-targeted patch-clamp neural recording in vivo. Neuron 95, 1037-1047.e10 (2017).

Annecchino, L.A. et al. Robotic automation of in vivo two-photon targeted whole-cell patch-clamp electrophysiology. Neuron 95, 1048-1055.e3 (2017). 